\title{
Construção de uma cartilha educativa acerca do processo cirúrgico pediátrico: experiência utilizando literatura de cordel
}

\author{
Construction of an educational booklet about the paediatric surgical process: experience using \\ cordel literature
}

Construcción de un folleto educativo sobre el proceso quirúrgico pediátrico: experiencia con la literatura de cordel

\author{
Bárbara Maria Viana de Couto \\ ORCID: https://orcid.org/0000-0001-6493-4126 \\ Universidade de Pernambuco, Brasil \\ E-mail: barbara.mvcouto@upe.br \\ Beatriz Mendonça Morais Alves \\ ORCID: https://orcid.org/0000-0003-4520-8588 \\ Universidade de Pernambuco, Brasil \\ E-mail: beatriz.mendonca @upe.br \\ Bruna Maiara Carvalho Correia da Silva \\ ORCID: https://orcid.org/0000-0002-5520-2748 \\ Universidade de Pernambuco, Brasil \\ E-mail: bruna.maiara@upe.br \\ Dhebora Santos de Souza \\ ORCID: https://orcid.org/0000-0003-0299-5219 \\ Universidade de Pernambuco, Brasil \\ E-mail: dhebora.santos@upe.br \\ Elyan Matheus Aguiar de Lima \\ ORCID: https://orcid.org/0000-0003-1129-5898 \\ Universidade de Pernambuco, Brasil \\ E-mail: elyan.matheus@upe.br \\ Joana D'arc Vila Nova Jatobá \\ ORCID: https://orcid.org/0000-0002-0881-4442 \\ Universidade de Pernambuco, Brasil \\ E-mail: joana.darc@upe.br \\ Waldemar Brandão Neto \\ ORCID: https://orcid.org/0000-0003-4786-9961 \\ Universidade de Pernambuco, Brasil \\ E-mail: waldemar.neto@upe.br
}

\begin{abstract}
Resumo
Objetivo: relatar o processo de construção de uma cartilha de orientação cirúrgica pediátrica em formato de cordel e sua importância para o protagonismo das crianças e de suas famílias durante a vivência cirúrgica. Método: trata-se de relato de experiência, desenvolvido por discentes e docentes de uma Universidade pública de Pernambuco, durante a vivência do ensino remoto no período de setembro à novembro de 2020. A construção foi realizada em quatro etapas: levantamento bibliográfico, elaboração do cordel, design da cartilha e avaliação. Resultados: a atividade resultou na cartilha em formato de cordel 'O dia da minha cirurgia", que facilita o entendimento das crianças e de suas famílias acerca do procedimento cirúrgico, e orienta de forma descontraída. Considerações finais: a cartilha em formato de cordel se configurou como recurso acessível, lúdico, criativo e didático, que dialoga com a cultura popular nordestina, podendo potencializar a educação popular em saúde, como estratégia de promoção à saúde no contexto hospitalar.

Palavras-chave: Procedimentos cirúrgicos operatórios; Comunicação em saúde; Literatura; Enfermeiras pediátricas.
\end{abstract}

\begin{abstract}
Objective: to relate the process of constructing a pediatric oriented cartilage in the form of a cordel and its importance for the protagonism of children and their families during surgical life. Method: This is an experience report, developed by students and teachers at a public university in Pernambuco, during the experience of remote teaching in the period from September to November 2020. The construction was carried out in four stages: bibliographic survey, preparation of the cordel, design of the booklet, and evaluation. Results: the activity resulted in the cordel-format booklet "The day of my surgery", which facilitates the understanding of children and their families about the surgical procedure, and provides guidance in a relaxed way. Final considerations: the cordel-format booklet was configured as an
\end{abstract}


accessible, playful, creative, and didactic resource, which dialogues with the popular culture of the Northeast, and can potentiate popular education in health, as a health promotion strategy in the hospital context.

Keywords: Surgical procedures, Operative; Health communication; Literature; Nurses, Pediatric.

\section{Resumen}

Objetivo: relatar el proceso de construcción de un cartílago de orientación pediátrica en forma de cordón y su importancia para el protagonismo de los niños y de sus familias durante la vida quirúrgica. Método: se trata de un relato de experiencia, desarrollado por discentes y docentes de una Universidad pública de Pernambuco, durante la vivencia del aprendizaje remoto en el período de septiembre a noviembre de 2020. La construcción se realizó en cuatro etapas: levantamiento bibliográfico, elaboración del cordón, diseño de la cartilla y evaluación. Resultados: La actividad dio como resultado el folleto en formato de cordel "El día de mi cirugía", que facilita la comprensión de los niños y sus familias sobre el procedimiento quirúrgico, y proporciona orientación de forma relajada. Consideraciones finales: la cartilla en formato de cordel se configuró como un recurso accesible, lúdico, creativo y didáctico, que dialoga con la cultura popular del Nordeste de Brasil, y puede potenciar la educación popular en salud, como estrategia de promoción de la salud en el contexto hospitalario.

Palabras clave: Procedimientos quirúrgicos operativos; Comunicación en salud; Literatura; Enfermeras pediátricas.

\section{Introdução}

A Literatura de Cordel, também chamada poesia popular impressa, trata-se de uma manifestação literária caracterizada pela impressão em folhetos e exposição em barbantes ou cordões - daí sua denominação, que teve origem na região da Península Ibérica e chegou ao Brasil através de colonizadores portugueses (Pagliuca, Oliveira, Rebouças, \& Galvão, 2007). Essas produções literárias geralmente retratam a vida de cangaceiros, lendas, mitos e estórias do povo sertanejo (S. Lima, 2011); entretanto, hodiernamente, também assumiram o papel de ferramenta didática, dado seu caráter lúdico e sua polivalência enquanto meio de comunicação em saúde e facilitador da construção de conhecimento (Pagliuca et al., 2007; Silveira, Alves, Bertoncici, \& Rodrigues, 2015). A Educação no campo da Saúde, amparada pela acepção freiriana, fundamenta-se na transição da consciência ingênua para a consciência crítica, na problematização da realidade, na dialogicidade e na valorização dos saberes populares (Freire, 2019). Consoante Freire (2019), os saberes têm naturezas distintas e, portanto, não se deve estimar a existência de saberes melhores ou piores.

Se observado sob a mesma ótica dos constructos epistemológicos de Freire, o cordel também atua como um instrumento metodológico que favorece a valorização dos saberes e da linguagem do povo. Partindo da premissa que a Educação em Saúde demanda, especialmente, uma abordagem simples e acessível, a poesia popular impressa potencialmente assume uma finalidade pedagógica por ser uma ferramenta de baixo custo, além de possuir sonoridade e utilizar expressões populares (Martins et al., 2011; Castro \& Costa, 2015).

Ademais, a Literatura de Cordel pode desempenhar o papel de tecnologia de educação para saúde, despertando a atenção dos leitores e contribuindo para a formação de uma consciência crítica e transformadora acerca do tema trabalhado (Pagliuca et al., 2007; Silveira et al., 2015; Martins et al., 2011). Este instrumento educativo fomenta a participação dos sujeitos e os situa enquanto protagonistas do processo, contribuindo para a autonomia dos indivíduos em conformidade com as teorias emancipatórias freirianas (Freire, 2019).

No tocante à aplicabilidade do cordel enquanto ferramenta educativa, existem diversos estudos que retratam a eficácia de tal recurso na prática. Temáticas como infecções sexualmente transmissíveis (Pagliuca et al., 2007), saúde bucal (Castro \& Costa, 2015), saúde do homem (Sousa, Capistrano, T. C. Oliveira, M. T. Oliveira, \& Costa, 2017), entre outras, quando trabalhadas por intermédio da Literatura de Cordel na qualidade de instrumento didático-pedagógico de Educação em Saúde, evidenciaram resultados promissores.

Ainda sob tal perspectiva, pode-se inferir a relevância da Educação em Saúde nos serviços hospitalares. No que se refere à assistência prestada por profissionais de enfermagem a crianças hospitalizadas, é evidente a importância do estabelecimento de vínculo entre a tríade criança-família-profissionais. Isso se dá porque tais estratégias de aproximação 
tornam a hospitalização menos traumatizante para a criança e para os cuidadores. Além disso, a utilização de recursos lúdicos incita a mitigação de sentimentos negativos inerentes ao processo de internação hospitalar, favorecendo o tratamento e consequente recuperação (Falke, Milbrath, \& Freitag, 2018). O período de hospitalização é permeado por diversas emoções negativas, tais como preocupação, medo, ansiedade, desconforto, impotência e angústia. Esses sentimentos também estão presentes e são potencializados quando a criança é submetida a procedimentos cirúrgicos (Costa, Nobre, Gomes, Nornberg, \& Rosa, 2019; Lima et al., 2019). Em pesquisa exploratória e descritiva (Broering \& Crepaldi, 2018), obteve-se que mais da metade das genitoras demonstraram preocupação e estresse ao serem informadas da necessidade da cirurgia. De acordo com o estudo, os principais sentimentos relatados pelas crianças que seriam submetidas ao procedimento cirúrgico foram medo e ansiedade. Ainda conforme essa pesquisa, foi possível reconhecer que a incipiência de informações sobre a doença e a cirurgia suscitaram sentimentos negativos nos familiares e nas crianças.

Destarte, é indiscutível a necessidade da comunicação efetiva entre a equipe de saúde, os cuidadores e a criança hospitalizada. Isso porque, havendo uma preparação, os pacientes e familiares sentem-se mais seguros quanto aos procedimentos, compreendem a situação e colaboram com o processo, almejando a recuperação da saúde da criança (Costa et al., 2019; Broering \& Crepaldi, 2018). Isso posto, constata-se que a Literatura de Cordel, além de se tratar de uma ferramenta de intensa penetração, é uma forma de expressão cultural que utiliza uma linguagem simples, acessível, além de estimular o imaginário dos indivíduos, o que a configura como um recurso auspicioso com vistas a ressignificar o cuidado em saúde (Martins et al., 2011; Castro \& Costa, 2015).

Diante do exposto, o presente estudo objetivou relatar a experiência sobre a construção de uma cartilha educativa utilizando-se da Literatura de Cordel a fim de informar pacientes e seus cuidadores acerca do processo cirúrgico pediátrico, de modo a minimizar impactos negativos por meio de uma comunicação lúdica e humanizada.

\section{Metodologia}

Trata-se de um estudo descritivo, com abordagem qualitativa, do tipo relato de experiência, caracterizado no âmbito das pesquisas que consideram a subjetividade dos processos, validado como produção científica a partir do alinho da narrativa experiencial com o embasamento teórico (Daltro \& Faria, 2019; Pereira, Shitsuka, Parreira, \& R. Shitsuka, 2018). O estudo apresenta a construção de material em formato de cartilha educativa, com objetivo de informar e orientar crianças e seus responsáveis acerca dos aspectos que compreendem os procedimentos em crianças submetidas à cirurgia. Este relato resulta das atividades de ensino remoto durante a pandemia da Covid-19, produzido por discentes e docentes do Módulo V Dimensão do cuidar III - Saúde da Criança e do Adolescente, da Faculdade de Enfermagem Nossa Senhora das Graças (FENSG) da Universidade de Pernambuco.

A proposta é justificada a partir do entendimento de que a hospitalização de crianças, principalmente para fins cirúrgicos, compreende diversos fatores estressantes. Estudos apontam a necessidade de reduzir a ansiedade dos responsáveis, entender o contexto da hospitalização de crianças e o impacto gerado nas famílias, e identificar as vulnerabilidades presentes em cada caso, de modo a prestar uma assistência de qualidade. (Akçay Didişen, Karakul, Ertürk, \& Dökümcü, 2020). Evidencia-se a necessidade de informação, uma vez que a literatura reforça o nível de instrução em saúde como sendo preditor da ansiedade dos pais e/ou responsáveis, além de ser um item considerado muito importante pelos mesmos durante o período perioperatório. (Kampouroglou et al., 2020; Akçay Didişen, Karakul, Ertürk, \& Dökümcü, 2020).

Sendo assim, compete ao profissional prestar assistência completa e de qualidade, exercendo a comunicação em saúde, a fim de elevar o nível de informação de pais e/ou responsáveis, bem como das próprias crianças em situação de hospitalização, com o propósito de reduzir altos graus de nervosismo e ansiedade, além de prevenir problemas decorrentes de quadros estressantes. Destarte, diversas ferramentas podem ser utilizadas para realizar educação em saúde, informando e 
orientando pessoas sobre as mais variadas temáticas. A escolha da literatura de cordel deu-se por ser uma ferramenta didáticopedagógica de baixo custo, fácil compreensão e capaz de alcançar distintos grupos. A assimilação e disseminação do conteúdo informativo são favorecidas na literatura de cordel por ser constituída de métrica, rimas, ritmo, sonoridade e imagens (Silva et al., 2010), traduzindo-se em um eficiente aliado nas práticas de educação popular em saúde.

A partir de tal compreensão sobre o tema, foi possível concretizar as intenções primordiais, dando início à sistematização da construção da cartilha expressa em cordel, organizando a produção nas seguintes etapas:

1. Levantamento bibliográfico: Os descritores em ciências da saúde (DeCS) que compuseram a estratégia de busca corresponderam à "Assistência Perioperatória", "Cuidados de Enfermagem" e "Enfermagem". Acrescentando a palavra-chave "Cirurgia pediátrica" foi possível realizar a busca no Portal Regional da BVS, obtendo como resultado 99 materiais bibliográficos. Os pesquisadores utilizaram a estratégia para efetuar também buscas independentes no Google Scholar. O processo de seleção dos estudos que serviram de base para a elaboração do cordel pode ser visualizado através da Figura 1.

Figura 1 - Fluxograma de seleção dos artigos incluídos na fase do levantamento bibliográfico.

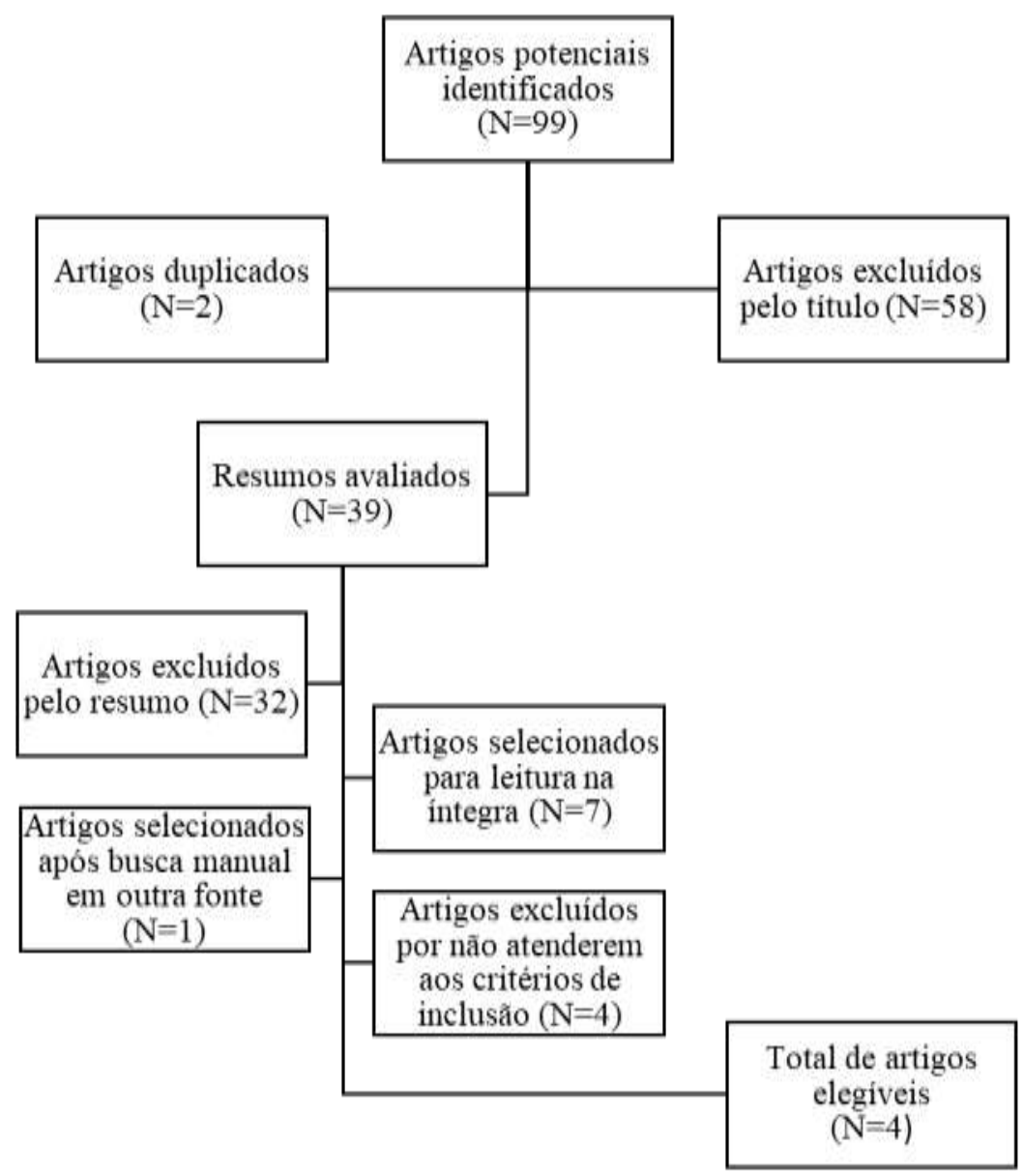

Fonte: Autores (Recife-PE, Brasil, 2020).

Observa-se, na Figura 1, o percurso traçado para o levantamento bibliográfico, contando primeiramente com uma triagem para artigos duplicados $(\mathrm{N}=2)$ e, posteriormente, para artigos excluídos pelo título $(\mathrm{N}=58)$ e pelo resumo $(\mathrm{N}=32)$. Percebe-se ainda que a avaliação da elegibilidade dos estudos prosseguiu com a leitura na íntegra de 8 artigos, sendo, portanto, 4 deles elegíveis para o propósito de conferir à cartilha parte do caráter informativo através das evidências científicas. Os artigos elegíveis abordaram a importância da assistência de enfermagem no perioperatório, a percepção de crianças hospitalizadas sujeitas às experiências cirúrgicas e os cuidados perioperatórios indispensáveis. 
2. Elaboração do cordel: Alguns pontos imprescindíveis sobre os cuidados perioperatórios foram elencados a partir do referencial teórico, sendo dispostos em ordem para facilitar o planejamento do cordel. Como ponto de partida, definiu-se que a criança sujeita à cirurgia seria a protagonista, de modo que a idealização para o cordel foi feita sob a perspectiva da criança que recebe uma assistência de qualidade no período perioperatório. Para fins de interatividade, um checklist foi adicionado ao cordel. Assim, a criança inclusa em seu processo terapêutico, pode marcar as ações como efetuadas, à medida em que os cuidados necessários forem sendo executados em seu determinado tempo. Por fim, o cordel foi intitulado "O dia da minha cirurgia".

3. Design da cartilha: Composta de três páginas, frente e verso, a cartilha hospeda capa, contracapa, e o cordel. Ao longo do cordel é possível identificar espaços “( )" para assinalar com um X. Além dos elementos verbais, foram acrescentados recursos gráficos (como figuras e traçados característicos de cordéis) à apresentação visual da cartilha, organizados por meio da utilização dos aplicativos de criação e design Paint Tool Sai® e PhotoScape ${ }^{\circledR}$. Tais elementos podem ser observados na Figura 2.

Figura 2 - Capa e contracapa da cartilha em cordel "O dia da minha cirurgia".
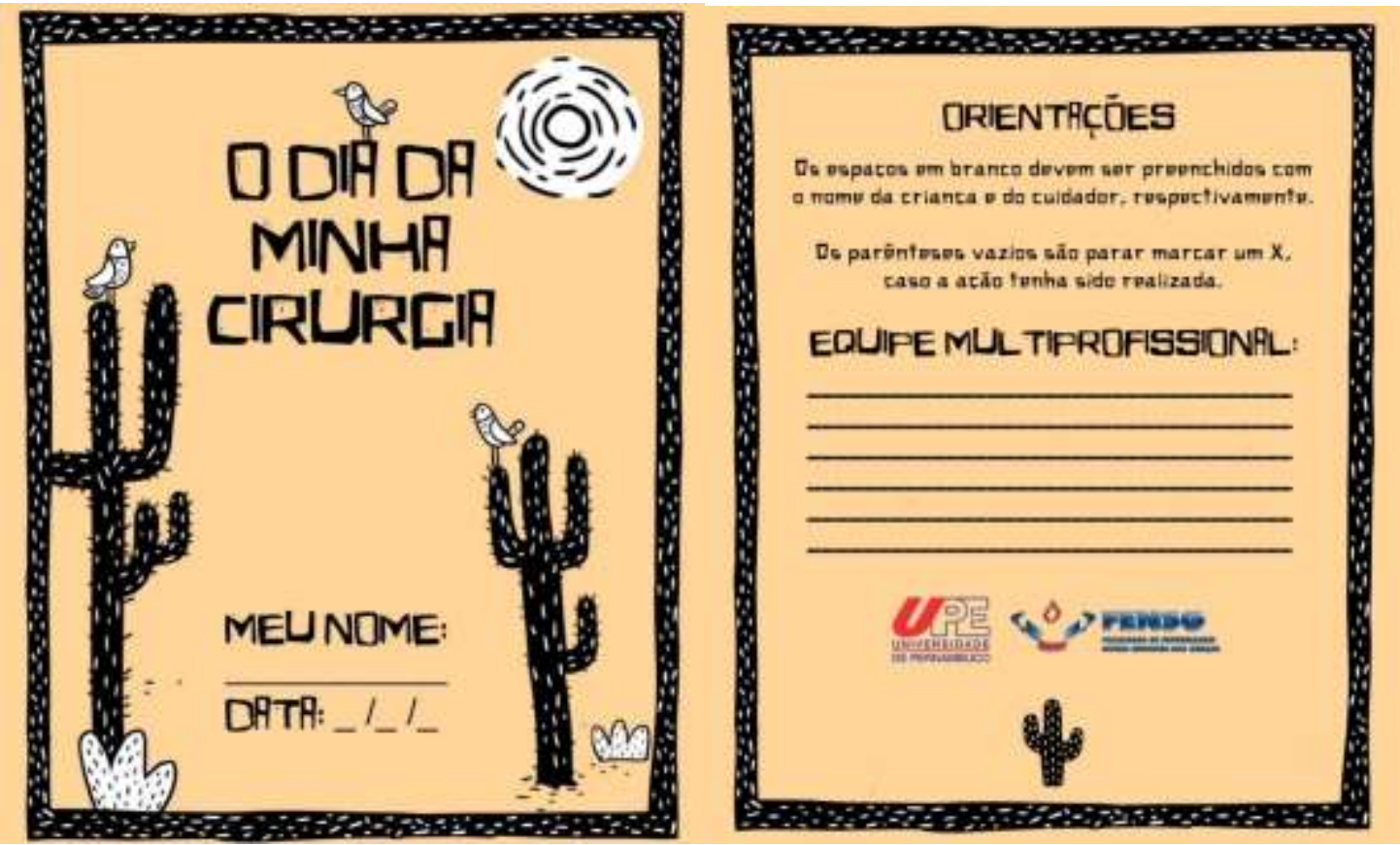

Fonte: Autores (Recife-PE, Brasil, 2020).

Na Figura 2, é possível notar a capa da cartilha contendo ilustrações em xilogravuras, título e espaços para acrescentar o nome da criança e a data de recebimento do material. Na contracapa, verificam-se as orientações para preenchimento, bem como nome da equipe multiprofissional e instituição de origem.

4. Fase de avaliação: A avaliação primária do material ocorreu mediante envio da cartilha para que os docentes do módulo pudessem analisar minuciosamente a qualidade do que foi produzido, de acordo com critérios previamente estabelecidos de conformidade com as evidências científicas, criatividade e viabilidade de aplicabilidade. Seguidamente, o produto foi apresentado pelo grupo, através de ferramentas tecnológicas de ensino remoto. A apresentação contou com uma introdução, explicação sobre o método utilizado para compor a cartilha e recitação do cordel. Em conclusão, os docentes avaliaram positivamente o trabalho exposto, destacando não só a relevância da literatura de cordel para a cultura nordestina, mas também a criatividade do grupo perante a temática e a importância de se ter na prática assistencial um material agregando 
o saber científico ao conhecimento popular, gozando assim de uma verdadeira potencialidade de educação popular em saúde.

Considerando o exposto, apresentando o estudo como um relato de experiência, descrevendo uma atividade de produção acadêmica, entende-se que não foi necessária avaliação pelo sistema CEP/CONEP, de acordo com o Art. $1^{\circ}$ da Resolução nº 510 de 07/04/2016, pelo expresso caráter exclusivo de educação, ensino ou treinamento do presente estudo.

\section{Resultados}

O produto final da resolução da atividade foi uma cartilha de abordagem didática e interativa, que utiliza componentes textuais e visuais com o objetivo de permitir a plena compreensão acerca das informações apresentadas.

A cartilha foi estruturada a partir de uma produção textual do gênero literário cordel, intitulada "O dia da minha cirurgia". O cordel foi constituído por onze estrofes setilhas, isto é, estruturadas em sete versos de sete sílabas poéticas, visto que essa modalidade favorece o cantar e a memorização do texto (Nóbrega, 2019). Os versos, por sua vez, apresentam rimas externas alternadas e interpoladas, tendo sido escritos em linguagem coloquial para tornar a composição acessível a distintos leitores.

O contexto de inserção do poema foi baseado no processo de hospitalização e internação para cirurgia pediátrica, discorrendo as etapas que permeiam o período operatório de maneira encadeada e utilizando das rimas para trazer ludicidade à descrição dos eventos. O cenário de ocorrência dos fatos é o hospital ou serviço de saúde e as personagens secundárias são o responsável da criança e a própria equipe multiprofissional de cuidados, trazendo ênfase para o profissional enfermeiro.

O texto ainda conta com uma abertura à definição da personagem principal, que deve ser a criança que está sendo submetida à cirurgia, adequando os eventos citados à realidade da mesma ou ao imaginário criado em torno da situação. Para esse fim, foram dispostos espaços para interação na própria estrutura do cordel, como lacunas a serem preenchidas no decorrer do escrito e assertivas sobre o processo de internação a serem constatadas e confirmadas pelo leitor, com o objetivo de gerar uma aproximação entre o poema e a realidade da criança hospitalizada.

Seguem trechos do cordel para fins de ilustração: "Meu acolhimento foi ótimo/ Tenho certeza que sou capaz/ Já estou por dentro de tudo/ E minha equipe é muito eficaz/ Agora é esperar o grande dia/ Pra quando eu fizer minha cirurgia/ E nada disso me incomodar mais [...] Mas também precisam da minha ajuda/ Por isso presto atenção!/ Para entender tudo que a enfermeira fala/ (E ela é ótima na explicação!)/ Me falou do jejum ( ) e da anestesia ( )/ Viu se tenho dentes moles ( ) ou alergia ( )/ Me explicou sobre os exames e a internação ( ) [...] Meu acolhimento foi ótimo/ Tenho certeza que sou capaz/ Já estou por dentro de tudo/ E minha equipe é muito eficaz/ Agora é esperar o grande dia/ Pra quando eu fizer minha cirurgia/ E nada disso me incomodar mais".

Além dos elementos verbais foram acrescentados recursos gráficos, como figuras e traçados característicos para a apresentação visual da cartilha, que foram organizados por meio da utilização de aplicativos de criação e design. Toda a composição da apresentação, desde as cores à fonte da letra utilizadas foram escolhidas no intento de remeter à cultura nordestina, visto que a literatura de cordel é uma expressão artística popular típica dessa região e a caracterização do material permite ao paciente a construção de um vínculo de identificação com o contexto do que se lê.

No que tange a oralidade da declamação do cordel, há a exigência de uma pronúncia específica e ritmada, que é inerente ao gênero literário. Todavia, por se tratar de uma obra que integra a expressão cultural popular regional, essa recitação apresenta-se de maneira simples e as rimas por si só norteiam o compasso do ritmo da leitura.

Isto posto, ressalta-se que toda a disposição dos componentes da cartilha foi elaborada tencionando a concepção de uma afinidade entre a criança e o processo de hospitalização perioperatório, mesclando ideias lúdicas e elementos culturais para empregar sutileza à vivência da internação. 


\section{Discussão}

A composição da cartilha educativa tem como desígnio oferecer ao profissional de saúde uma ferramenta lúdica que conduza tanto a criança, em sua particularidade, quanto seus familiares, sobre o período perioperatório, com propósito de minimizar as dificuldades enfrentadas, especialmente, no pré-operatório e na admissão no centro cirúrgico. Assim, a literatura de cordel educa para a promoção da saúde, principalmente, porque permite a integração dos saberes populares ao saber científico e pode ser utilizada não só na instrução de crianças, como também de jovens e adultos (Pagliuca et al. 2007; Silveira et al., 2015; Martins et al., 2011; Castro \& Costa, 2015).

Englobado especialmente pela enfermagem, o material aspira propiciar a educação adequada à família e ao público infantil - foco leitor dos folhetos - de forma a contribuir para a devida compreensão por ambos os grupos. As informações fornecem uma visão técnica geral acerca do perioperatório em cirurgia pediátrica. Outrossim, a leitura também pode ser proveitosa para profissionais da saúde, sob a perspectiva de uma orientação acerca de quais tipos de intervenções estes podem realizar, além da estimulação à criatividade do profissional.

A comparência de acompanhantes adequadamente habilitados a auxiliar a criança mostra-se enquanto componente essencial de uma experiência pré-operatória menos estressante, uma vez que a ansiedade dos cuidadores está relacionada funcionalmente ao nível de estresse dos pacientes (Akçay Didişen et al., 2020; Mello, Moraes, \& Costa Junior, 2019). Dados de um estudo nacional quanto aos efeitos da informação em crianças descrevem que a experiência da cirurgia se torna menos estressante quando pacientes e cuidadores adequadamente habilitados participam do contexto hospitalar (Mello et al., 2019). Os níveis de ansiedade dos pais se correlacionam com os níveis de dor pré e pós-operatória das crianças, o que sugere a importância de ludoterapias com capacidade de reduzir tais comportamentos na criança, com a diminuição dos efeitos também percebidos nos pais (Yayan, Zengin, Düken, \& Suna Dağ, 2020).

Oferecer suporte emocional a esses cuidadores, através de um material educativo relacionado ao processo de internação e aos cuidados necessários durante esse período, pode fazer desta cartilha informativa em literatura de cordel uma proveitosa e acessível fonte de informação. Pesquisadores em um estudo chileno constataram que os responsáveis relatam a necessidade e desejam informações além das verbais sobre a cirurgia e o ambiente cirúrgico, pois muitos sentem medo e ansiedade antes da cirurgia de um filho, o que dificulta sua preparação para o procedimento (Sartori et al., 2015). Muitas vezes, esta expectativa dos pais não converge com a ação dos enfermeiros, por desconhecerem o conceito e a aplicabilidade das terapias lúdicas e atividades do brincar em ambiente hospitalar, reforçando a necessidade de suscitar discussões e experimentações destas tecnologias educativas na graduação, como uma possibilidade dentro do Processo de Enfermagem (Berté et al., 2017).

Acerca dos critérios que se revelam convenientes para conduzir o conteúdo disponibilizado ao público em questão, podem ser referidos a idade e o nível de desenvolvimento cognitivo da criança, bem como a disponibilidade antecipatória do material educativo, que, por razões financeiras e logísticas, tende a ser oferecido apenas no dia da execução do procedimento cirúrgico. Ainda assim, o protagonismo infantil no contexto hospitalar, atrelado de um material guia suporte, visa tornar a criança uma agente de seu aprendizado, e não alguém simplesmente passivo na educação. Para isso, são levadas em consideração suas necessidades, suas limitações e seu ritmo para o pleno funcionamento do processo de ensino e aprendizagem.

Para reforçar a importância da literatura de cordel como um instrumento didático-pedagógico estimulador na educação, motivação e promoção em saúde, um estudo nacional realizado em uma instituição pública de ensino de Natal - RN, sobre saúde bucal, utilizando cordéis que foram confeccionados acerca dessa temática em saúde e recitados para escolares de 8 a 13 anos, afirmaram que os conhecimentos prévios foram acrescidos ou modificados após a intervenção literária (Castro \& Costa, 2015). Nesse sentido, o cordel se incorpora como recurso educativo, considerando que a educação requer especialmente, 
uma linguagem simples, acessível e adequada a cada público (Martins et al., 2011; Castro \& Costa, 2015). Pôde-se perceber através da leitura e interpretação dos significados das falas que a literatura de cordel causou um impacto no processo de aprendizagem e no comportamento do imaginário de cada criança, o que só amplia a validação de sua aplicação como instrumento em saúde, considerando que o lúdico torna o aprendizado mais leve e prazeroso.

Adicional à temática, outro estudo nacional, realizado na região do semiárido baiano, que descreve a experiência na elaboração e utilização da literatura de cordel como estratégia para a educação popular em saúde de homens, destaca que a criação de uma cartilha informativa em saúde para a população masculina pode não só proporcionar a reflexão sobre a situação e o engajamento na busca pelo enfrentamento dos agravos, mas também contribuir de modo efetivo para a redução da morbidade e mortalidade dessa população por meio do enfrentamento racional dos fatores de risco (Sousa et al., 2017). A literatura de cordel, por constituir-se em um veículo importante de interlocução e comunicação de maneira expressiva, é um elemento relevante para educação, com poder para estimular práticas de autocuidado, e assim, promover saúde (Pagliuca et al. 2007; Silveira et al., 2015; Martins et al., 2011).

É consentâneo salientar que, por vezes, instrumentos educativos literários podem não ser tão instigantes à curiosidade para o aprendizado lúdico do público infantil, em contrapartida a outros meios e estratégias educativas que abrangem conteúdos gráficos, tais como vídeos, animações e jogos. Não obstante, destaca-se a relevância do folheto de cordel como um recurso de suporte para as crianças e seus responsáveis, permitindo a revisão das orientações em todo o período de espera que venha a anteceder a realização da cirurgia pediátrica. Ainda, ressalta-se a importância da distribuição desse material educativo de modo gratuito aos pacientes e seus acompanhantes, enquanto artigo impresso e sustentado em uma linguagem simples, a fim de tornar a comunicação acessível.

Ao percurso do desenvolvimento deste trabalho, identificaram-se limitações importantes quanto aos achados de pesquisas e artigos acadêmicos atuais referentes à temática da construção de livretos e cartilhas educativas para a orientação do período perioperatório pediátrico. Acrescenta-se também, como limitação do estudo, o fato de que a cartilha construída ainda não foi submetida a um processo de validação de aparência e conteúdo. Apenas a validação do material por um grupo de profissionais poderá confirmar ou sugerir modificações que tornarão a cartilha eficiente. Estudos metodológicos consideram que as análises de instrumentos na área da saúde devem ser multiprofissionais, e que, após sua aprovação, o instrumento poderá contribuir para novos processos intervencionais em educação de saúde para os posteriores público-alvo (Leite et al., 2018).

A partir do exposto, esse estudo contribui para uma discussão sobre os papéis dos profissionais de saúde, em especial do enfermeiro, na assistência pediátrica, que devem ir além da realização de protocolos clínicos. Por fim, foi possível inferir que o recurso lúdico é fundamental tanto para o desenvolvimento das habilidades cognitivas e sociais, quanto para dirimir o efeito das experiências desagradáveis provocadas pela hospitalização e afastamento de suas atividades sociais.

\section{Considerações Finais}

Este relato de experiência revelou que é possível a construção de uma cartilha de orientação cirúrgica pediátrica em formato de cordel, capaz de fortalecer vínculos e reduzir sentimentos negativos que surgem no período perioperatório. Como material acessível, lúdico, criativo e didático, que dialoga com a cultura popular nordestina, a cartilha em formato de cordel pode ser um recurso fortalecedor da educação popular em saúde, como estratégia de promoção à saúde no contexto hospitalar.

A obtenção de resultados positivos mediante o uso do cordel nos poucos estudos encontrados e utilizados como base para o presente artigo transparecem a necessidade da produção de mais materiais que favoreçam a utilização desse método informativo, a fim de disseminar seu uso na prática assistencial dos serviços de saúde em pediatria.

Quanto à estrutura conceitual, metodológica e as intenções da cartilha, alguns pontos merecem ser destacados: 
reafirmar o direito da criança à informações sobre sua saúde, o estímulo ao protagonismo da criança na experiência cirúrgica, participação da família, autonomia da Enfermagem na utilização de ferramentas lúdicas como adjuvante na clínica cirúrgica pediátrica, além do processo interdisciplinar alcançado com a experiência, por meio da apropriação da linguagem literária e a valorização dos saberes populares.

Assim, a cartilha intitulada "O dia da minha cirurgia" pode ser aperfeiçoada com potencial para aumentar a confiança da criança e pais no procedimento, qualificação da comunicação no ambiente hospitalar e satisfação tanto do paciente e sua família quanto da equipe de saúde. Ademais, a possibilidade de trabalhar recursos lúdicos para aperfeiçoar o cuidado à criança, permite fornecer segurança ao futuro profissional em experimentar práticas viáveis e de baixo custo em seu ambiente de trabalho, pensando o cordel como uma possibilidade de cuidar em enfermagem pediátrica.

Outrossim, destacamos que fatores como tempo e a realidade do ensino remoto, ocasionado pela pandemia da Covid19, inviabilizaram o uso da cartilha cirúrgica nos serviços hospitalares. Tem-se como próximo passo do estudo, diante de uma mudança no cenário atual, a apresentação da cartilha para a equipe de enfermagem, pacientes e familiares, com posterior realização de um estudo de validação e inferir os possíveis benefícios em unidades pediátricas após avaliação dos recursos e capacitação do enfermeiro pediátrico.

Por fim, faz-se necessário o desenvolvimento de estudos de intervenção baseado em ferramentas lúdicas com o cordel no contexto hospitalar para avaliar o impacto nos seguintes desfechos críticos da pediatria: a antecipação da expectativa da dor; a percepção de perigo; os medos relacionados aos procedimentos e instrumentos cirúrgicos; a perda de controle; as rotinas e pessoas desconhecidas; a ansiedade; e a separação temporária dos pais, de modo a contribuir com práticas assistenciais humanizadas ao paciente pediátrico.

\section{Referências}

Akçay D. N., Karakul, A., Ertürk, S., \& Dökümcü, Z. (2020). Anxiety Levels and Needs of Fathers of Children Hospitalized in Pediatric Surgery Intensive Care Units. The journal of pediatric research, 7(1), 38-45. 10.4274/jpr.galenos.2019.83435

Berté, C., Ogradowski, K. R. P., Zagonel, I. P. S., Tonin, L., Favero, L., \& Almeida Junior, R. de. L. (2017). Brinquedo Terapêutico No Contexto Da Emergência Pediátrica. Revista baiana de enfermagem / Universidade Federal da Bahia, Escola de Enfermagem, 31(3), 1-10. 10.18471/rbe.v31i3.20378

Broering, C., \& Crepaldi, M. A. (2018). Percepções e informações das mães sobre a cirurgia de seus filhos. Fractal: revista de psicologia, 30(1), 3-11. $10.22409 / 1984-0292 / \mathrm{v} 30 \mathrm{i} 1 / 1434$

Castro, M. C. de S., \& Costa, I. do C. C. (2015). A literatura de cordel como instrumento didático-pedagógico na educação, motivação e promoção da saúde bucal. Revista Ciência Plural, 1(1), 40-49. https://periodicos.ufrn.br/rcp/article/view/7322

Costa, A. R., Goulart Nobre, C. M., Gomes, G. C., Nornberg, P. K. de O., \& Rosa, G. S. M. (2019). Sentimentos gerados na família pela internação hospitalar da criança. Journal of nursing and health / Faculdade de Enfermagem UFPEL, 9(2), 1-12. 10.15210/jonah.v9i2.14012

Daltro, M. R., \& Faria, A. A. de (2019). Relato de experiência: uma narrativa científica na pós-modernidade. Estudos e Pesquisas em Psicologia, 19(1), 223237. http://pepsic.bvsalud.org/scielo.php?script=sci_arttext\&pid=S1808-42812019000100013

Falke, A. C. S., Milbrath, V. M., \& Freitag, V. L. (2018). Estratégias Utilizadas Pelos Profissionais Da Enfermagem Na Abordagem a Criança Hospitalizada. Revista Contexto \& Saúde, 18(34), 9-14. 10.21527/2176-7114.2018.34.9-14

Freire, P. (2019). Pedagogia do Oprimido. (75a ed.). Paz \& Terra.

Kampouroglou, G., Velonaki, V. S., Pavlopoulou, I., Drakou, E., Kosmopoulos, M., Kouvas, N., Tsagkaris, S., Fildissis, G., Nikas, K., \& Tsoumakas, K. (2020). Parental anxiety in pediatric surgery consultations: the role of health literacy and need for information. Journal of pediatric surgery, 55(4), 590-596. 10.1016/j.jpedsurg.2019.07.016

Leite, S. de S., Áfio, A. C. E., Carvalho, L. V. de, Silva, J. M. da, Almeida, P. C. de, \& Pagliuca, L. M. F. (2018). Construction and validation of an Educational Content Validation Instrument in Health. Revista brasileira de enfermagem, 71(suppl 4), 1635-1641. 10.1590/0034-7167-2017-0648

Lima, R. M., Gomes, F. M. A., Aguiar, F. A. R., Santos Junior, E. B. Dos., Dourado, J. V. L., \& Ferreira Junior, A. R. (2019). Experiências de mães durante a internação hospitalar de seus filhos. Revista de pesquisa, cuidado é fundamental online, 11(5), 1286. 10.9789/2175-5361.2019.v11i5.1286-1292

Lima, S. (2011). A pedagogia do cordel. Delicatta. 
Research, Society and Development, v. 10, n. 3, e38110313379, 2021

(CC BY 4.0) | ISSN 2525-3409 | DOI: http://dx.doi.org/10.33448/rsd-v10i3.13379

Martins, A. K. L., Nunes, J. M., Nóbrega, M. de F. B., Pinheiro, P. N. da C., Souza, A. M. A. E., Vieira, N. F. C., \& Fernandes, A. F. C. (2011). Literatura de cordel: tecnologia de educação para saúde e enfermagem. Revista enfermagem UERJ [internet], 19(2), 324-329. https://pesquisa.bvsalud.org/portal/resource/pt/lil-601594

Mello, S. T. T. de, Moraes, L. U. De., \& Costa Junior, Á. L. (2019). Crianças e procedimentos cirúrgicos: efeitos da informação. Revista Psicologia \& Saúde [Internet], 11(3), 97-109. 10.20435/pssa.v11i3.701

Nóbrega, J. (2019). Literatura De Cordel: Uma proposta didática analisando a tradição de 10 décadas (1912 a 2013). Revista Encontros de Vista, 23(1), 40-51. http://www.journals.ufrpe.br/index.php/encontrosdevista/article/view/4137

Pagliuca, L. M. F., Oliveira, P. M. P., Rebouças, C. B. de A., \& Galvão, M. T. G. (2007). Literatura de cordel: veículo de comunicação e educação em saúde. Texto \& contexto enfermagem / UFSC, 16(4), 662-670. 10.1590/s0104-07072007000400010

Pereira, A. S., Shitsuka, D. M., Parreira, F. J., \& Shitsuka, R. (2018). Metodologia da Pesquisa Científica UFSM.

Sartori, J., Espinoza, P., Díaz, M. S., Ferdinand, C., Lacassie, H. J., \& González, A. (2015). ¿Qué información preoperatoria desean los padres de niños que serán operados? Revista chilena de pediatría, 86(6), 399-403. 10.1016/j.rchipe.2015.06.021

Silva, S. P. Da., Arcanjo, J. G., Souza, H. C. B. De., Silva, R. M. S., Souza, C. O. De., Lucena, C. S. De., Araújo, W. E. De., Lucena, K. G. M. De., \& Tenório, A. C. (2010). Literatura de cordel: linguagem, comunicação, cultura, memória e interdisciplinaridade. Revista Raído [Internet], 4(7), 303-322. https://ojs.ufgd.edu.br/index.php/Raido/article/view/603/539

Silveira, J. L. G. C., Alves, M. U., Bertoncici, J. H., \& Rodrigues, K. F. (2015). Literatura de cordel como estratégia em educação popular em saúde. Revista de atenção primária saúde: APS.[Internet], 18(4), 532-538. https://periodicos.ufjf.br/index.php/aps/article/view/15711

Sousa, A. R., Capistrano, R. de L., Oliveira, T. C. de, Oliveira, M. T., \& Costa, M. S. F. (2017). Cordel como estratégia de educação popular na saúde de homens. Revista de Educação Popular, 16(1), 140-155. http://www.seer.ufu.br/index.php/reveducpop/article/view/35964

Yayan, E. H., Zengin, M., Düken, M. E., \& Suna Dağ, Y. (2020). Reducing Children's Pain and Parents’ Anxiety in the Postoperative Period: A Therapeutic Model in Turkish Sample. Journal of pediatric nursing, 51, e33-e38. 10.1016/j.pedn.2019.07.004 